\title{
Erradicación del Helicobacter pylori en pacientes obesos pre-cirugía bariátrica
}

\author{
Oscar Laudanno, ${ }^{1}$ Gabriel Ahumarán, ${ }^{2}$ Marcelo Thomé, ${ }^{3}$ Pablo Gollo, ${ }^{2}$ Marina Khoury ${ }^{4}$ \\ ${ }^{1}$ Departamento de Gastroenterología, Instituto de Investigaciones Médicas "Alfredo Lanari”, Universidad de Buenos Aires. Ciudad Autónoma de \\ Buenos Aires, Argentina. \\ 2 Sección Gastroenterología, Hospital Bocalandro. Tres de Febrero, prov. de Buenos Aires, Argentina. \\ ${ }^{3}$ Departamento de Gastroenterología, Hospital Eva Perón. San Martín, prov. de Buenos Aires, Argentina. \\ ${ }^{4}$ Docencia e Investigación, Instituto de Investigaciones Médicas "Alfredo Lanari”. Universidad de Buenos Aires, Ciudad Autónoma de Buenos Aires, \\ Argentina.
}

Acta Gastroenterol Latinoam 2020;50(1):40-44

Recibido: 25/02/2019 / Aceptado: 12/03/2019 / Publicado online: 23/03/2020 / https://doi.org/10.52787/ksmb4439

\section{Resumen}

Introducción. Muchos grupos especializados en cirugía bariátrica recomiendan la búsqueda de rutina del Helicobacter pylori (H. pylori) como parte de la evaluación pre-cirugía bariátrica. Algunos estudios sugieren que los pacientes obesos tienen una baja tasa de erradicación y que el Indice de Masa Corporal podría ser un factor de riesgo independiente. Objetivo. Evaluar diferencias en la tasa de erradicación del H. pylori con un esquema cuádruple concomitante por 14 dias en pacientes obesos pre-cirugía bariátrica comparado

Correspondencia: Oscar Miguel Oreste Laudanno Combatientes de Malvinas 3150 (C.P.: 1427). Departamento de Gastroenterología, Instituto de Investigaciones Médicas "Alfredo Lanari", Universidad de Buenos Aires. Ciudad Autónoma de Buenos Aires, Argentina / Tel.: +54(011) 52873849

Correo electrónico: oscarlaudanno@gmail.com con un grupo control. Material y métodos. Estudio prospectivo, que incluyó a 76 pacientes obesos pre-cirugía bariátrica y 75 pacientes controles con IMC normal y dispepsia. Resultados. La erradicación del H. pylori fue del 69\% en el grupo de pacientes obesos mientras que en el grupo control fue del 88\%. $p<0,05$, Odds ratio de 3, 18 (IC 1,35-7,45). La distribución de edad, género, fumadores, diabetes no difirió significativamente entre ambos grupos. Conclusiones. Los pacientes obesos pre-cirugía bariátrica tienen una significativa menor tasa de erradicación del $H$. pylori que pacientes con IMC normal con un esquema cuádruple concomitante de 14 dias.

Palabras claves. Helicobacter pylori, obesidad, cirugia bariátrica, endoscopia preoperatoria.

\section{Eradication of Helicobacter pylori in Obese Pre-Bariatric Surgery Patients}

\section{Summary}

Introduction. Many bariatric surgeons recommend routine Helicobacter pylori (H. pylori) screening as a part of the workup for bariatric surgery. Some studies suggested that obese patients have a lower eradication rate and the Body Mass Index could be independent risk factor for this fail- 
ure. Objective. To evaluate the H. pylori eradication of a 14 days Quadruple Concomitant therapy in obese patients undergoing bariatric surgery compared against a control group. Material and Methods. Prospective study included 76 patients undergoing bariatric surgery and 75 control patients with a normal BMI and dyspepsia. Results. In the obese group, the eradication rate was $69 \%$ whereas in the control group was $89 \%$. $p<0.05$, Odds ratio 3.18 (CI 1.35-7.45). The distribution of age, gender, smoking and diabetes did not differ significantly between the two groups. Conclusions. Obese patients showed a significantly lower eradication of $H$. pylori than controls to a two weeks Quadruple Concomitant therapy.

Keywords. Helicobacter pylori, obesity, bariatric surgery, preoperative endoscopy.

\section{Introducción}

La Organización Mundial de la Salud (OMS) ha declarado a la obesidad como el problema de salud crónico en adultos más grande del mundo, con proporciones epidémicas en los países industrializados. Es responsable de muchas enfermedades crónicas, como las cardiovasculares y la diabetes, y genera grandes gastos de salud así como aumenta la mortalidad. ${ }^{1-3}$ La cirugía bariátrica es el tratamiento más efectivo para la reducción de peso a largo plazo, con mejoría de las comorbilidades, incremento de la calidad de vida y disminución de la mortalidad. Los procedimientos más habituales incluyen la gastrectomía en manga (Sleeve Gastrectomy) y el By pass en $Y$ de Roux (RYGB). 4, 5

La prevalencia de Helicobacter pylori (H. pylori) en pacientes derivados para cirugía bariátrica es variable, se ha reportado desde el 20 al 70\%, y depende en gran medida de la prevalencia en cada región. ${ }^{6-9}$ Los datos en cuanto a la relación entre el $H$. pylori, la obesidad y las complicaciones postoperatorias son controversiales. ${ }^{10-14}$ La ASGE (American Society for Gastrointestinal Endoscopy) y la ASMBS (American Society for Metabolic and Bariatric Surgery) recomiendan el testeo y erradicación del $H$. pylori pre-cirugía bariátrica en forma individualizada. ${ }^{15}$ Las guías europeas recomiendan el testeo de H. pylori previo a todas las cirugías bariátricas. ${ }^{16,17}$

En la Argentina, la mayor parte de los grupos quirúrgicos recomiendan la realización de videoendoscopía alta con búsqueda de $H$. pylori de rutina previo a la cirugía bariátrica, y su erradicación de ser positivo. ${ }^{18,19}$ La justificación de esta recomendación se basa en la posibilidad de síntomas digestivos altos postoperatorios así como de úlceras marginales o cáncer de estómago en el estómago remanente y la inaccesibilidad luego del By pass gástrico a la porción del estómago excluida. ${ }^{20-22}$

Las guías de tratamiento del $H$. pylori, como la de Maastricht $\mathrm{V}$, recomiendan esquemas cuádruples concomitantes y sugieren prolongar el tiempo de tratamiento de erradicación a 14 días en regiones con alta resistencia a claritromicina como es la Argentina. ${ }^{23,24}$ Si bien hay estudios que sugieren que los pacientes obesos tienen una baja tasa de erradicación, hay autores que plantean que la tasa de erradicación es similar. ${ }^{25,}{ }^{26}$ No encontramos estudios comparativos, con grupo control, que evaluaran el efecto del Índice de Masa Corporal (IMC) sobre la erradicación del $H$. pylori con esquemas cuádruples concomitantes prolongados.

\section{Objetivo}

Evaluar diferencias en la tasa de erradicación del $H$. pylori con un esquema cuádruple concomitante por 14 días en pacientes obesos pre-cirugía bariátrica comparado con un grupo control.

\section{Material y métodos}

Estudio prospectivo que incluyó a 76 pacientes consecutivos obesos pre-cirugía Bariátrica, y a 75 pacientes consecutivos con IMC normal y dispepsia. Todos los pacientes incluidos eran $H$. pylori positivos por examen histopatológico, las muestras fueron tomadas por videoendoscopía alta con sedación entre diciembre de 2014 y noviembre de 2016 en los hospitales C. Bocalandro y Eva Perón. Se excluyeron las pacientes embarazadas, en lactancia, con antecedente de alergia a alguna de las drogas utilizadas, con intento de erradicación previa del $H$. pylori, que recibieron tratamiento con antibióticos, inhibidores de bomba de protones o antagonistas $\mathrm{H} 2$ en las 4 semanas previas. Todos los pacientes fueron tratados con un esquema cuádruple concomitante con Pantoprazol 40 mgr, Amoxicilina 1gr, Claritromicina 500 mgr y Metronidazol 500 mgr, 2 veces al día, durante 14 días. El estudio fue aprobado por el Comité de Revisión Institucional del hospital y seguido de acuerdo a la Declaración de Helsinki. La determinación de la erradicación del $H$. pylori fue realizada 6-8 semanas luego del tratamiento mediante el test del aire espirado con ${ }^{13} \mathrm{C}$-Urea (Tau-Kit, Bacon). La adherencia y efectos adversos fueron evaluados al final del tratamiento mediante entrevista directa y autorreporte por el paciente. Los eventos adversos fueron categorizados como ausentes, leves cuando no interfirieron con la vida diaria, moderados cuando interfirieron con la vida diaria pero no impidieron el tratamiento y severos cuando llevaron a discontinuar el tratamiento. 
Análisis estadístico: los datos fueron analizados estadísticamente con el software OpenEpi. Para la comparación se utilizó el Test de chi cuadrado con corrección de Yates. Se consideró una $p<0,05$ como estadísticamente significativa. Se calculó el Odds Ratio (OR) y su Intervalo de Confianza (IC) para el 95\%.

\section{Resultados}

En cuanto a las características demográficas y antecedentes, ambos grupos de pacientes fueron homogéneos en cuanto a sexo, edad, proporción de fumadores y diabéticos (Tabla 1).
La tasa de erradicación del $H$. pylori fue del 69,7\% (53/76) en el grupo de pacientes pre-cirugía bariátrica con un IC $95 \%$ de $58-78$ y del $88 \%(66 / 75)$ en el grupo control con un IC del 95\% de 78-93 ( $p=0,01088)$. El Odds ratio fue de 3,182 (IC 1,359-7,454).

Un 16\% (24/151) de los pacientes manifestaron eventos adversos, los cuales fueron considerados leves ya que no interfirieron con la vida diaria y no llevaron a una discontinuación temprana. Estos fueron en su mayoría: gusto metálico $(n=6)$, diarreas $(n=4)$, dolor abdominal $(\mathrm{n}=4)$ y cefaleas $(\mathrm{n}=3)$. Otros menos frecuentes fueron náuseas, vómitos, distensión. Estos desaparecieron una vez finalizado el tratamiento.

Tabla 1. Descripción demográfica y comparación de los grupos

\begin{tabular}{|c|c|c|c|}
\hline Variable & $\begin{array}{l}\text { Grupo control } \\
\qquad(n=75)\end{array}$ & $\begin{array}{l}\text { Pacientes pre-cirugía bariátrica } \\
\qquad(\mathrm{n}=76)\end{array}$ & Valor $p$ \\
\hline $\begin{array}{l}\text { Sexo femenino } \\
n(\%)\end{array}$ & $43(57,3)$ & $40(52,6)$ & $p>0,05$ \\
\hline $\begin{array}{l}\text { Edad } \\
\text { media (rango) }\end{array}$ & $48,1(20-56)$ & $42,8(19-52)$ & $p>0,05$ \\
\hline $\begin{array}{l}\text { Fumadores } \\
n(\%)\end{array}$ & $24(32)$ & $20(26)$ & $p>0,05$ \\
\hline $\begin{array}{l}\text { Diabéticos } \\
n(\%)\end{array}$ & $10(13)$ & $14(18)$ & $p>0,05$ \\
\hline $\begin{array}{l}\text { IMC } \\
\text { mediana (rango) }\end{array}$ & $22(19-24)$ & 44 (35-52) & $p<0,05$ \\
\hline
\end{tabular}

\section{Discusión}

En la Argentina, los pacientes obesos en plan de cirugía bariátrica realizan una videoendoscopía alta con búsqueda de $H$. pylori como parte de su evaluación preoperatoria de rutina. Aquellos positivos se les recomienda la erradicación previa a la cirugía para disminuir las posibilidades de complicaciones post-operatorias, así como para prevenir el desarrollo de otras, esto cobra mucha relevancia en el By pass gástrico donde el estómago remanente queda excluido para su evaluación y es inaccesible por los métodos endoscópicos habituales. Es por ello que se considera importante que el paciente sea $H$. pylori negativo. Pero hay estudios que sugieren que los pacientes obesos tienen una menor tasa de erradicación y que el IMC es el factor de riesgo para no lograr la erradicación. La controversia persiste dado que hay pocos estudios que comparen la erradicación del $H$. pylori entre obesos y no obesos. Para nuestro conocimiento, hay un solo estudio que compare la tasa de erradicación del $H$. pylori en pacientes obesos con pacientes con IMC normal, y fue realizado con un esquema triple y de 7 días de duración, e incluyó pacientes con sobrepeso. Sus conclusiones fueron que los pacientes con sobrepeso/obesos tienen una baja tasa de erradicación. ${ }^{25}$ Por otro lado, hay autores como Pellicano ${ }^{27}$ que plantean que la tasa de erradicación es similar con base en un estudio realizado por Cerqueira y col., prospectivo, observacional, donde los pacientes obesos pre-cirugía bariátrica alcanzaron tasas de erradicación comparable a no obesos luego de 2 regímenes de $1^{\mathrm{a}}$ y $2^{\mathrm{a}}$ línea de tratamiento de acuerdo con lo sugerido por el Consenso de Maastricht IV. ${ }^{28}$ Pero este estudio adolece de un grupo de pacientes con IMC normal. Para Pellicano, el problema 
es la resistencia a los antibióticos y esta no es distinta en pacientes con IMC normal. El aporte del presente estudio fue mostrar que, con un esquema cuádruple concomitante de 14 días de duración acorde a un área con alta resistencia a claritromicina, los pacientes obesos en plan de cirugía bariátrica tienen una tasa de erradicación de $H$. pylori significativamente menor que pacientes con un IMC normal. Es por ello que creemos que la obesidad es un factor importante para la no erradicación.

Aunque los motivos de la diferencia no resultan claros, algunas explicaciones a esta menor erradicación en obesos podrían estar relacionadas con cambios en la farmacocinética y farmacodinamia de los antibióticos en individuos con incremento del tejido adiposo. La claritromicina y el metronidazol son drogas lipofílicas, pueden tener una baja concentración plasmática como resultado de un gran volumen de distribución. Las drogas hidrofílicas, como la amoxicilina, pueden tener afectada su concentración plasmática por cambios en el clearence renal con la hiperfiltración descripta en etapas iniciales, aunque puede haber disminución de la eliminación en etapas más avanzadas (glomerulopatía del paciente obeso). Es de remarcar que esta interacción entre la obesidad y la farmacocinéticafarmacodinamia de las drogas es compleja y muchas veces difícil de predecir ya que la magnitud de estos cambios es variable y depende de las características de los pacientes obesos (grado de obesidad, disfunción de algún órgano) así como de las propiedades de los fármacos. ${ }^{29-31}$

La mayor parte de los fármacos que utilizamos han sido desarrollados y testeados en personas con normopeso; las personas con obesidad han sido excluidos de la mayor parte de los ensayos clínicos. Es posible que el paradigma actual basado en dosis fijas para todos deba ser reemplazado por dosis individualizadas según el peso del individuo. . $^{32-34}$

Por otro lado, hay estudios epidemiológicos que sugieren que los pacientes obesos tienen mayor incidencia y severidad de infecciones además de una pobre respuesta antígeno-anticuerpo. La respuesta inmune estaría alterada y en parte se debería a la resistencia a la leptina. La leptina interviene en la respuesta de los linfocitos T. ${ }^{35}$

Otro posible mecanismo podría ser el retardo en la evacuación gástrica secundario a la ingesta mayor en grasas y el estilo de vida sedentario y esto conllevaría a una absorción menor de las drogas. ${ }^{36}$

En conclusión, en pacientes pre-cirugía bariátrica, un esquema cuádruple concomitante sin bismuto presentó una menor tasa de erradicación de $H$. pylori. Para mejorar los resultados consideramos necesario un enfoque más individualizado de dosis de fármacos basados en el IMC o regímenes terapéuticos distintos.
Sostén financiero. Este manuscrito no ha recibido financiación para su realización.

Conflicto de intereses. Los autores declaran no tener conflictos de interés.

Aviso de derechos de autor

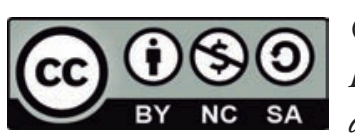

(C) 2021 Acta Gastroenterológica Latinoamericana. Este es un artículo de acceso abierto publicado bajo los términos de la Licencia Creative Commons Attribution (CC BY-NC-SA 4.0), la cual permite el uso, la distribución $y$ la reproducción de forma no comercial, siempre que se cite al autor y la fuente original.

Cite este artículo como: Laudanno $O$, Ahumarán $G$, Thomé $M$ y col. Erradicación del Helicobacter pylori en pacientes obesos pre-cirugia bariátrica. Acta Gastroenterol Latinoam. 2020;50(1):40-4. https://doi.org/10.52787/ ksmh4439

\section{Referencias}

1. Frühbeck G, Toplack H, Woodward E, Yumuk V, Maislos M, Oppert JM; Executive Committee of the European Association for the Study of Obesity. Obesity: the gateway to ill health-an EASO position statement on a rising public health, clinical, scientific challenge in Europe. Obesity Facts 2013; 6: 117-120.

2. Brolin R. Update: NIH consensus conference. Gastrointestinal Surgery for severe obesity. Nutrition 1996; 12: 403-404.

3. Calle E, Thun M, Petrelli J, Rodríguez C, Heath C. Body-mass index and mortality in a prospective cohort of U.S. adults. N Engl J Med 1999; 341: 1097-1105.

4. Klein S, Wadden T, Sugerman H. AGA Technical review on obesity. Gastroenterology 2002; 123: 882-932.

5. Yumuk V, Tsigos C, Fried M, Schindler K, Busetto L, Micic D, Toplak H. European Guidelines for Obesity Management in Adults. Obesity Facts 2015; 8: 402-424.

6. Erim T, Cruz-Correa MR, Szomstein S, Velis E, Rosenthal R. Prevalence of Helicobacter pylori seropositivity among patients undergoing bariatric surgery: a preliminary study. World J Surg 2008; 32: 2021-2025.

7. Verma S, Sharma D, Kanwar P, Sohn W, Mohanty S, Tortolani A, Gorecki P. Prevalence of Helicobacter pylori infection in bariatric patients: a histologic assessment. Surg Obes Relat Dis 2013; 9: 679-685.

8. Hartin CW Jr, ReMine DS, Lucktong TA. Preoperative bariatric screening and treatment of Helicobacter pylori. Surg Endosc 2009; 23: 2531-2534.

9. Papasayas P, Gagné D, Donnelly P, Salgado J, Urbandt J, Burton K, Caushaj P. Prevalence of Helicobacter pylori infection and value of preoperative testing and treatment in patients undergoing laparoscopic Roux-en-Y gastric bypass. Surg Obes Relat Dis 2008; 4: 383-388. 
10. Serin K, Akyüz Ü, Batman B, Uymaz D, Altun H. Does Helicobacter pylori infection influence the major postoperative complication rate after sleeve gastrectomy? A retrospective cohort study in an endemic region. Turk J Gastroenterol 2018; 29: 379-383.

11. Danciu M, Simion L, Poroch V, Paduireanu S, Constantinescu R, Arhire L, Mihalache L. The role of histological evaluation of Helicobacter pylori infection in obese patients referred to laparoscopic sleeve gastrectomy. Rom J Morphol Embryol 2016; 57: 1303-1311.

12. Rasmussen JJ, Fuller W, Ali MR. Marginal ulceration after laparoscopic gastric bypass: an analysis of predisposing factors in 260 patients. Surg Endosc 2007; 21: 1090-1094.

13. Smelt H, Smulders J, Gilisen L, Said M, Ugale S, Pouwels S. Influence of Helicobacter pylori infection on gastrointestinal symptoms and complications in bariatric surgery patients: a review and meta-analysis. Surg for Obes and Relat Dis 2018; 14: 1645-1657.

14. Peker K, Sabbaz N, Sevit H, Kopes O, Gumusogly A, Alis H. An alternative view on the necessity of EGD before sleeve gastrectomy. Surg Obes Relat Dis 2017; 13: 1959-1964.

15. Evans J, Muthusamy V, Acosta D, Bruining D, Chandrasekhara V, Chathadi K, Eloubeidi M, Fanelli R, Faulx A, Fonkalsrud L, Khashab M, Lightdale J, Pasha S, Saltzman J, Shaukat A, Wang A, Stefanidis D, Richardson W, Kothari S, Cash B, American Society for Gastrointestinal Endoscopy Standards of Practice Committee. The role of endoscopy in the bariatric surgery patient. Gastrointestinal Endoscopy 2015; 81: 1063-1072.

16. Fried M, Hainer V, Basdevant A, Buchwald H, Deitel M, Finer N, Greve J, Horber F, Mathus-Viegen E, Scopinaro N, Steffen R, Tsigos C, Weiner R, Widhalm K. Interdisciplinary European Guidelines on Surgery of Severe Obesity. Obesity Facts 2008; 1: 52-59.

17. De Palma G, Forestieri P. Role of endoscopy in the bariatric surgery of patients. World J Gastroenterology 2014; 20: 7777-7784.

18. Pampillón N, Reynoso C, Pagano C. Consenso Argentino de Nutrición en Cirugía Bariátrica. Actualización en Nutrición 2012; 12: 98-141.

19. Pampillón N, Reynoso C, Solimano M. Actualización del Consenso Argentino de Nutrición en Cirugía Bariátrica. Actualización en Nutrición 2016; 17: 19-32.

20. Batista Dantas C, Santo M, De Cleva R, Aissar Sallum R, Cecconello I. Influence of obesity and bariatric surgery on gastric cáncer. Cancer Biol Med 2016; 13: 269-276.

21. Schulman A, Abougergi M, Thompson C. H. pylori as Predictor of Marginal Ulceration: A Nationwide Analysis. Obesity 2017; 25: 522-526.

22. Carabotti M, D’Ercole C, Iossa A, Corazziari E, Slecchia G, Severi C. Helicobacter pylori infection in obesity and its clinical outcome after bariatric surgery. World J Gastroenterology 2014; 20: 647-653.
23. Malfertheiner P, Megraud F, Morain C, Gisbert J, Kuipers E, Taxon A, Bazzoli F, Gasbarrini A, Atherton J, Graham D, Hunt R, Moayyedi P, Rokkas T, Rugge M, Selgrad M, Suerbaum S, Sugano K, El-Omar E. Management of Helicobacter pylori infection-the Maastricht V/Florence Consensus Report. Gut 2017; 66: 6-30.

24. Molina Infante J, Corti R, Doweck J, McNicholl A, Gisbert J. Acta Gastroenterol Latinoam 2017; 47: 75-85.

25. Abdullahi M, Annibale B, Capoccia D, Tari R, Lahner E, Osborn J, Leonetti F, Severi C. The eradication of Helicobacter pylori is affected by body mass index (BMI). Obesity Surgery 2008; 18 : 1450-1454.

26. Cuesta Hernández M, Pérez Peña C, Matía Martín P, Cabrerizo García L, Perz-Ferre N, Sánchez Pernaute A, Torres García A, Rubio Herrera M. Helicobacter pylori $(H p)$ infection in obese patients undergoing Roux-en-Y gastric bypass; efficacy of two different treatment regimens in $H p$ eradication. Nutrición Hospitalaria 2015; 32: 600-605.

27. Pellicano R. Similar Helicobacter pylori Eradication Rate in Obese Patients Undergoing Gastric Bypass Surgery and in General Population. Obesity Surgery 2018; 28: 553-554.

28. Cerqueira R, Correira M, Vilar H, Manso M. Cumulative Helicobacter pylori Eradication rates by Adopting Fisrt-and Second-Line Regimens Proposed by Maastricht IV Consensus in Obese Patients Undergoing Gastric Bypass Surgery. Obesity Surgery 2017; 28: 743-747.

29. Hanrahan T, Lipman J, Roberts J. Antibiotic dosing in obesity: a BIG challenge. Critical Care 2016; 20: 240.

30. Anshika K, Adil M. Drug Dosing in Obese Patients: A dilemma. International Journal of Advances in Pharmaceutics 2014; 3: 59-65.

31. Barras M, Legg A. Drug dosing in obese adults. Australian Prescriber 2017; 40: 189-193.

32. Serra Soler G, Delgado Sánchez O, Martínez Lopez I, Pérez Rodríguez $\mathrm{O}$. Recomendaciones para la dosificación de fármacos en la obesidad mórbida. Medicina Clínica (Barcelona) 2008; 130: 778-782.

33. Hanley M, Abernethy D, Greenblat D. Effect of obesity on the phamacokinetics of drugs in humans. Clin Pharmacokinet 2010; 49: 71-87.

34. Pintar T, Kaliterna N, Carli T. The need for a patient-tailored Helicobacter pylori eradication protocol prior to bariatric surgery. Journal of International Medical Research 2018; 46: 2696-2707.

35. Marti M, Marcos A, Martínez J. Obesity and immune function relationships. Obes Rev 2011; 2: 131-140.

36. Jackson S, Leahy F, McGowan A, Bluck L, Coward W, Jebb S. Delayed gastric emptying in the obese: an assessment using the non-invasive ${ }^{13} \mathrm{C}$-octanoic acid breath test. Diabetes Obes Metab 2004; 6: 264-270. 